\title{
Real Time Self-Tuning Controller for Position Control of DC Motor System using Pole-Placement Technique
}

\author{
Mohammad Tarik Mohammad \\ Computer Engineering Department, \\ College of Engineering, \\ Alternative Location \\ Center of University of \\ Mosul at Kirkuk City, Iraq \\ Allaa Zaki \\ Computer Engineering Department, \\ College of Engineering, \\ Alternative Location \\ Center of University of \\ Mosul at Kirkuk City, Iraq
}

\author{
Sara Basim \\ Computer Engineering Department, \\ College of Engineering, \\ Alternative Location \\ Center of University of \\ Mosul at Kirkuk City, Iraq \\ Maher Algreer \\ School of Electrical and \\ Electronic Engineering, \\ Newcastle University, \\ Newcastle upon Tyne, UK
}

\begin{abstract}
This paper presents a real time self-tuning controller for DC motor system. Pole-placement adaptive controller based on Exponentially Recursive Least Square (ERLS) algorithm is proposed. The parameters of the DC motor are estimated using ERLS algorithm. Once the estimation error is minimized, the identified parameters are forward to the supervisory control unit to find the corresponding PID gains. A PCI-6251 data acquisition card from National Instrument (NI) and the prototyped control system (33-100 \& 33-110) from feedback device are deployed for real time implementation of the proposed solution. Simulation and experimental results verified the effectiveness of the ERLS algorithm, where the parameters of DC motor are estimated rapidly and accurately. Results, also shows the validation of the proposed self-tuning controller for position control of the DC motor system.
\end{abstract}

\section{General Terms}

Control Algorithm.

\section{Keywords}

Real time control, DC Motor, Pole-placement controller, ERLS Algorithm, System identification

\section{INTRODUCTION}

Many of industrial control systems are widely using the DC motors in their applications [1]. For instance, robotic systems, electrical vehicles, electrical cranes, etc [1]. This is due to the simplicity of control design, reliability and linearity of DC motor [1,2]. In this regard, great consideration should be attained to develop a highly dynamic performance controller. Commonly, the PID controller with constant gains values is used for controlling the DC motors. This is because the PID controller is able to improve both static and dynamic characteristic of the plant, as well as it is simple to implement and tune [3-5]. In order to successfully tune the PID gains, an accurate model of the controlled system is required [6]. This can be achieved via system identification algorithms; two paradigms are considered: parametric method and nonparametric method [7]. As presented in the literature, a nonadaptive PID controller is less successful for controlling the DC motor, where some of system parameters are uncertain or unknown such as external load torque, disturbance etc. [2, 8]. From this, intelligent controllers such as adaptive and selftuning controller received special attention in DC motor drive applications [2,9]. These types of controllers are capable to improve the overall response of the controlled system [10]. Different techniques are presented in the literature, for instance the authors in $[11,12]$ apply the fuzzy controller approach for digitally control of DC motor, while in [13-16] a neural network controller is proposed. Model reference method and adaptive techniques are also demonstrated in [9, $17,18]$. In this paper, an alternative scheme of adaptive controller for positing control of the DC motor is proposed. The presented controller is simpler than many of the state-ofthe art methods, and it can be easily integrated with other control approaches, also it is appropriate for real time operation.

\section{DISCRETE TIME MODELLING OF THE DC-SERVO MOTOR \& ERLS ALGORITHM}

\subsection{Servo Motor Modelling}

The first step in parametric system identification is to define the discrete model of the plant [7]. Many of the systems can mathematically be described, while others are derived experimentally based on the observed input-output data. Here, the proposed identification scheme is applied to upon the armature model of the DC motor (Fig .1). Based on derived differential equations, the transfer function from the input voltage to the motor displacement can easily be described as [19]:

$$
\frac{\theta_{m}(s)}{E_{a}(s)}=\frac{K_{m}}{L_{a} J_{m} s^{3}+\left(R_{a} J_{m}+B_{m} L_{a}\right) s^{2}+\left(K_{b} K_{m}+R_{a} B_{m}\right) s}
$$

Where, $e_{a}(t)$ is the armature voltage, $L_{a}$ is the armature inductor, $R_{a}$ is the armature resistance, $J_{m}$ is the rotor inertia, $B_{m}$ the viscous frictional coefficient and $\theta_{m}(t)$ is the rotor displacement. 


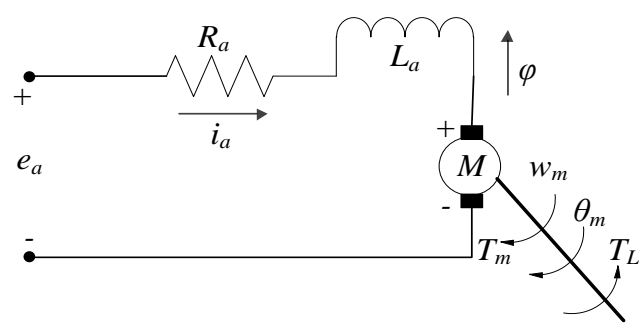

Fig.1. Armature DC Motor Model

Now, the continuous-time transfer function described in (1) can be converted to a discrete equivalent model using zerohold discrete transformation method, resulting in a third-order discrete transfer function:

$$
G_{\theta E}(z)=\frac{b_{1} z^{-1}+b_{2} z^{-2}+b_{3} z^{-3}}{1+a_{1} z^{-1}+a_{2} z^{-2}+a_{3} z^{-3}}
$$

Here, $b_{1}, b_{2}, b_{3}, a_{1}, a_{2}$, and $a_{3}$ are the lumped parameters to be estimated. The relation between the input-output may also be defined by using a linear difference equation. In this work, the best candidate model is the Autoregressive Moving Average (ARMA) model:

$$
\theta_{m}(n)=\sum_{k=0}^{M} b_{k} e_{a}(n-k)+\sum_{k=1}^{N} a_{k} \theta_{m}(n-k)
$$

Where, the data and parameters vectors are defined as follow:

$$
\begin{aligned}
\boldsymbol{\varphi} & =\left[\begin{array}{llllll}
-\theta_{m}(n-1) & \ldots & -\theta_{m}(n-k) & e_{a}(n-1) & \ldots & e_{a}(n-k)
\end{array}\right]^{T} \\
\boldsymbol{\Omega} & =\left[\begin{array}{llllll}
a_{1} & \ldots & a_{n} & b_{1} & \ldots & b_{n}
\end{array}\right]^{T}
\end{aligned}
$$

From (3) and (4), the estimated model can be directly described as:

$$
\widehat{\theta}=\varphi^{T} \Omega
$$

\subsection{Recursive Least Square Algorithm (RLS)}

In system identification and adaptive controller, it is important to estimate the system model and update the control parameters quickly and accurately [7]. Among other existing methods, the literature shown that RLS algorithms offer a simple, accurate, fast convergence time method, as well as it is directly applied in on-line and real time adaptive controller [3]. The RLS algorithm is processed the measured inputoutput data iteratively till the desired objective function is minimized (ideally to zero), indicating optimal parameters estimation is achieved [7]:

$$
J(n)=\sum_{k=1}^{n} e_{s}^{2}(k)=\sum_{k=1}^{n}\left[\theta_{m}(k)-\mathbf{\Omega}^{T} \boldsymbol{\varphi}(k)\right]^{2}
$$

The exponential recursive least square (ERLS) algorithm is summarized as follow [20]:

$$
\mathbf{S}(n)=\mathbf{P}(n-1) \varphi(n)
$$

$$
\mathbf{k}(n)=\frac{\mathbf{S}(n)}{\lambda+\varphi^{T}(n) \mathbf{S}(n)}
$$

$$
\begin{aligned}
& e(n)=\theta_{m}(n)-\hat{\mathbf{\Omega}}^{T}(n-1) \boldsymbol{\varphi}(n) \\
& \hat{\mathbf{\Omega}}(n)=\hat{\mathbf{\Omega}}(n-1)+\mathbf{k}(n) e(n) \\
& \mathbf{P}(n)=\frac{1}{\lambda}\left[\mathbf{P}(n-1)-\mathbf{k}(n) \boldsymbol{\varphi}^{T}(n) \mathbf{P}(n-1)\right]
\end{aligned}
$$

Where, , $\boldsymbol{\varphi}(n)$ is the data vector, $\boldsymbol{\Omega}(n)$ is the parameters vector, $e(n)$ estimation error, $\mathbf{P}(n)$ is the covariance matrix, $\mathbf{k}(n)$ is the adaptation gain vector, and $\lambda$ is the forgetting factor. In order to effectively track and monitor the variation of the parameters within the system, the forgetting factor should be varied between $0<\lambda<1$; at $\lambda=1$ the algorithm is known as classical RLS. It is important mentioning that high value of $\lambda$ improves the convergence time, but on expense of high sensitivity to noise and disturbances, whereas at low value of $\lambda$ the algorithm is less sensitive to noise, however the parameters relatively takes long time to converge to the desired values [7].

\section{SYSTEM SIMULATION AND VALIDATION}

This section presents the DC motor model simulation and the on-line validation of ERLS algorithm. The following parameters of the DC motor are used [19]: $L_{a}=0.56 \mathrm{mH}, R_{a}=$ $1.35 \Omega, J_{m}=0.0019 \mathrm{~kg} . \mathrm{m}^{2}, B_{m}=0.000792 \mathrm{~kg} . \mathrm{m}^{2} / \mathrm{s}, K_{b}=0.10$ $\mathrm{V} / \mathrm{rad} / \mathrm{s}$, and $K_{m}=0.1 \mathrm{~N} . \mathrm{m} / \mathrm{A}$. Now, from substituting the aforementioned values in (1), the discrete open loop transfer function of the DC motor can be determined as:

$$
G_{\theta E}(z)=\frac{0.04475 z^{-1}+0.043 z^{-2}+5.144 \times 10^{-6} z^{-3}}{1-1.806 z^{-1}+0.8056 z^{-2}}
$$

Equivalently, (12) can be approximated to second order model as follow:

$$
G_{\theta E}(z)=\frac{0.04475 z^{-1}+0.043 z^{-2}}{1-1.806 z^{-1}+0.8056 z^{-2}}
$$

For a normal closed loop operation of the position DC motor system, a digital PD controller is inserted in the feed-forward path of the closed loop system (Fig. 2):

$$
C_{P D}(z)=\frac{U_{p d}}{E(z)}=K_{p}+K_{D} \frac{z-1}{z-\alpha}
$$

The gains of the PD controller are tuned using trial and error method as: $K_{P}=0.5, K_{D}=2.5$, and $\alpha=0.1$. A 5-bit Pseudo Random Binary Sequence (PRBS) is injected into the control loop to excited the output signal and improve the convergence rate [7]. Now, the ERLS algorithm coefficients are initialized as follow: $\lambda=0.95, \boldsymbol{\Omega}=0, \mathbf{P}(0)=\frac{1}{\delta} \mathbf{I}$. $\mathbf{I}$ is the identity matrix, and $\delta$ is a constant value, usually $\delta$ is very low value. As shown in Fig. 2, we initially assumed that the system is working under normal closed loop operation using the digital PD controller (14). Once the identification process is activated, the PRBS is injected into the feed-back loop superimposed with the control action signal (Fig .2). In this simulation, the PRBS is injected for $20 \mathrm{sec}$ during the steadystate period. It has been found that this time is sufficiently enough for ensuring converges of the estimated parameters. However, the algorithm can also be started at the initial startup of the closed loop system, as well as it can be worked continuously into the feedback loop. The magnitude of the PRBS is selected to be as small as adequately cause an 
exaction into the output response $\left(\Delta_{P R B S}=0.3\right)$. Importantly, the injected amplitude is not influenced the typical operation of DC motor.

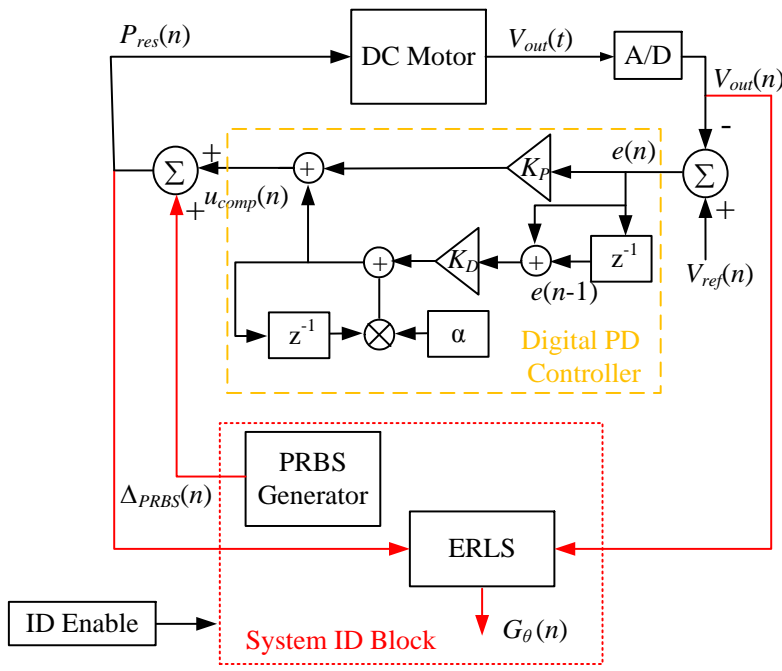

Fig.2. Closed loop identification based on RLS algorithm.

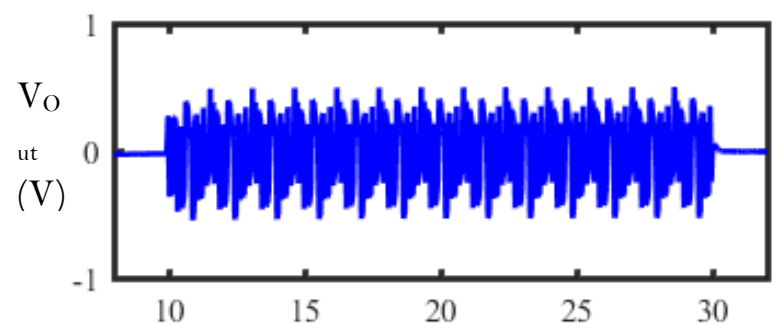

(a), Time(s)

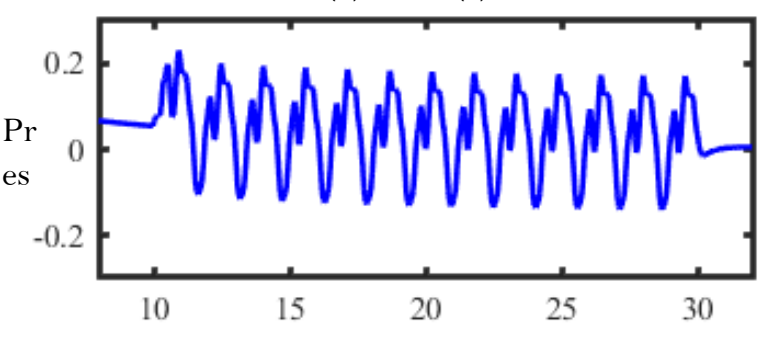

(b), Time(s)

Fig.3. Zero- mean: (a): Output voltage. (b): Persistence exaction signal.

Now, the ERLS algorithm starts iteratively to identify the system parameters and minimizing the estimation error amplitude. This process is accomplished by observing the zero mean value of the input and output signal on sampled basis (Fig. 3). As a result, the ERLS algorithm estimates the parameters of the DC motor, which is equivalent to the parameters of (13). Fig. 4 verifies the success of the proposed algorithm. Note that the ERLS quickly identify the parameters of DC motor; this in turn leads to minimize the estimated error signal (Fig. 5, a). These results are also confirmed in Fig. 5, b. As presented in Fig. 7, the ERLS algorithm estimated the parameters of the DC motor with a convergence rate less than $4 \mathrm{~s}$, at a sampling rate equal to $50 \mathrm{~ms}$. From the obtained results, we can confirm the capability and the effectiveness of utilizing the ERLS algorithm in real time adaptive control system.

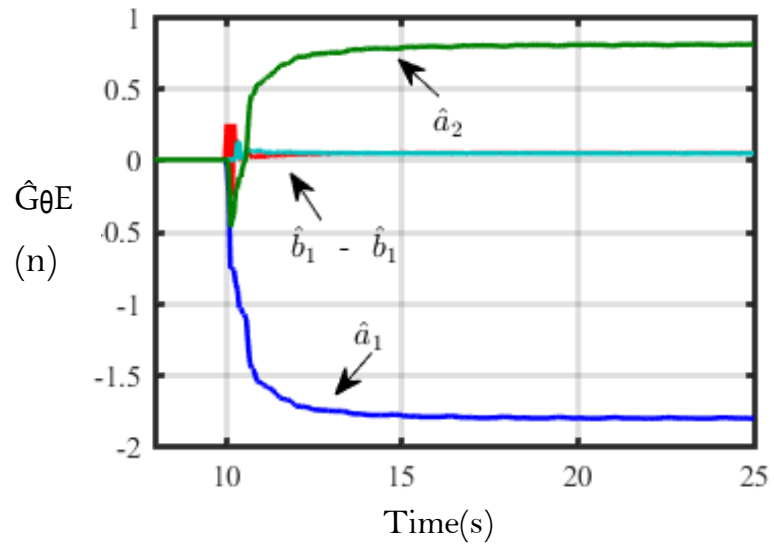

Fig.4. Parameters estimation of DC Model

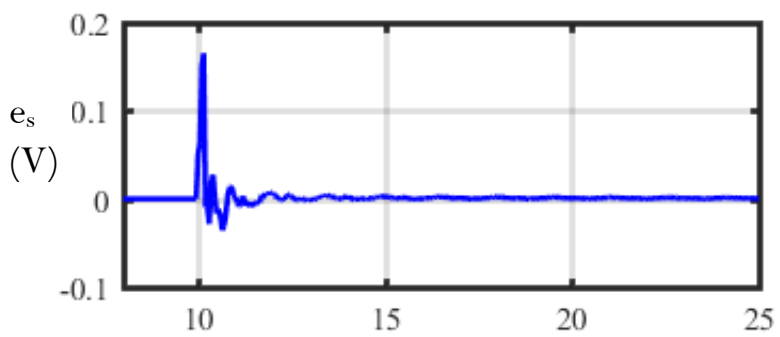

(a), $\operatorname{Time}(\mathrm{s})$

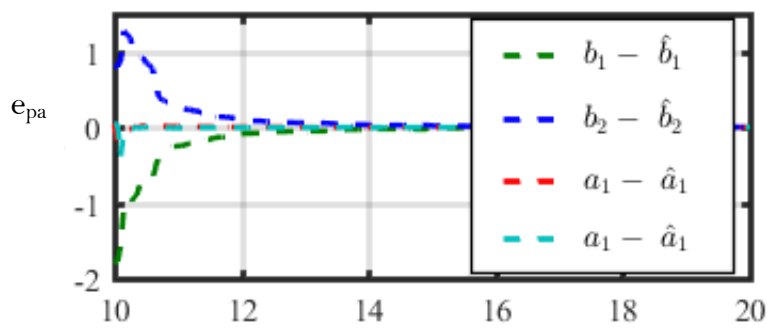

(b), Time(s)

Fig.5. (a): Estimation error signal. (b): Parameters estimation error

\section{ADAPTIVE POLE PLACEMENT}

\section{CONTROLLER}

Pole placement controller is considered as a direct adaptive control structure. In this approach, the coefficients of the controller are tuned continuously reliant directly on estimated parameters. In this project, a two poles/two zeros discrete PID controller will be deployed for the digital control of the DC motor system (15) [21]. Importantly, the order of the PID controller is based on the order of the plant (16).

$$
\begin{aligned}
& G_{c}(z)=\frac{\beta_{o}+\beta_{1} z^{-1}+\beta_{2} z^{-2}}{\left(1-z^{-1}\right)\left(1+\alpha z^{-1}\right)}=\frac{\beta(\mathrm{z})}{\alpha(\mathrm{z})} \\
& G_{\theta E}(z)=\frac{B(z)}{A(z)}=\frac{b_{1} z^{-1}+b_{2} z^{-2}}{1+a_{1} z^{-1}+a_{2} z^{-2}}
\end{aligned}
$$

As shown in Fig. 6, the closed loop control transfer function can be written as follow [21, 22]:

$$
G_{L}(z)=\frac{B(z) \beta(z)}{A(z) \alpha(z)+B(z) \beta(z)}
$$




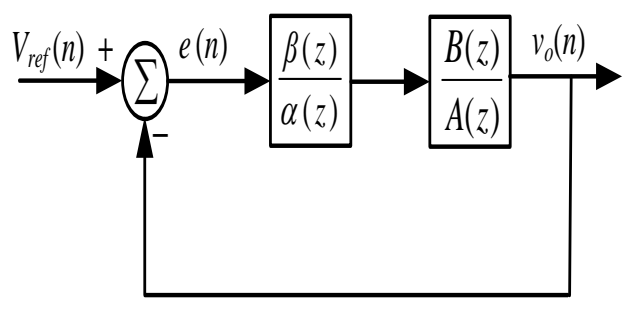

Fig.6. Closed loop control of the DC motor.

The desired closed loop dynamic of the system can be used to solve the relation in the denominator polynomial expressed in (17). In this way, the locations of the closed loop poles are set according to the desired values, unlike other control techniques which required tuning of the control coefficients for acceptable response [21] .

$$
\begin{aligned}
D(z) & =A(z) \alpha(z)+B(z) \beta(z)=1+\sum_{k=1}^{N_{d}} d_{k} z^{-k} \\
& =1+d_{1} z^{-1}+d_{2} z^{-2}+\cdots+d_{N_{d}} z^{-N} d, N_{d} \leq 4
\end{aligned}
$$

For simplicity, a second order characteristic equation is often utilised to describe the desired closed loop dynamics of the system [21]:

$$
G(s)=s^{2}+2 \xi w_{n}+w_{n}^{2}=0
$$

Therefore, the dynamic characteristic of a closed loop control may be given as in (20) [21]. As presented in (19) the dynamics behaviour is defined by the damping factor and the natural frequency. These factors should be selected appropriately for better performance and adequate damping response:

$$
\begin{aligned}
& d_{1}=-2 e^{-\xi w_{n} T_{s}} \cos \left(w_{n} T_{s} \sqrt{1-\xi^{2}}\right) \\
& d_{2}=e^{-2 \xi w_{n} T_{s}}
\end{aligned}
$$

To determine the parameters of the control system, the sets of linear algebra equations are required. This can be obtained by rewriting equation (18) in matrix form (21) [21]. Thus, the parameters of the PID controller can be solved as described in (22).

$$
\begin{aligned}
& {\left[\begin{array}{cccc}
b_{1} & 0 & 0 & 1 \\
b_{2} & b_{1} & 0 & a_{1}-1 \\
0 & b_{2} & b_{1} & a_{2}-a_{1} \\
0 & 0 & b_{2} & -a_{2}
\end{array}\right]\left[\begin{array}{c}
\beta_{0} \\
\beta_{1} \\
\beta_{2} \\
\alpha
\end{array}\right]=\left[\begin{array}{c}
d_{1}+1-b_{1} \\
d_{2}+b_{1}-b_{2} \\
b_{2} \\
0
\end{array}\right], N_{d}=2} \\
& \beta_{o}=\frac{1}{b_{1}}\left(d_{1}+1-a_{1}-\alpha\right), \beta_{1}=\frac{a_{2}}{b_{2}}-\beta_{2}\left(\frac{b_{1}}{b_{2}}-\frac{a_{1}}{a_{2}}+1\right), \beta_{2}=-\frac{s}{r} \\
& \alpha=\beta_{2} \frac{b_{2}}{a_{2}}, s=a_{2}\left(\left[b_{1}+b_{2}\right]\left[a_{1} b_{2}-a_{2} b_{1}\right]+b_{2}\left[b_{1} d_{2}-b_{2} d_{1}-b_{2}\right]\right) \\
& r=\left[b_{1}+b_{2}\right]\left[a_{1} b_{1} b_{2}+a_{2} b_{1}{ }^{2}+b_{2}{ }^{2}\right]
\end{aligned}
$$

Form (21) and (22), it can clearly be noticed that the design of the pole placement control action required the prices knowledge of the process parameters. This can be accomplished by the mean of system identification. Here, the adaptive pole-placement technique is developed based on ERLS algorithm. The ERLS identified the process parameters at each time instant; accordingly, the parameters of the control are updated continually. Once the optimized parameters of the model are estimated, therefore the estimation error is minimized, thus the effective control loop is achieved.

\section{EXPERIMENTAL}

\section{IMPLEMENTATION And RESULTS}

Fig. 7 (a, b) presents the block diagram of the proposed selftuning controller and the prototyped system setup. For sake of rapid test, a data acquisition card (NI-6521) is used to measure the input and output signals at each sample rate from the prototype servomechanism system (feedback device/33$100 \& 33-110)$. Note that the parameters of the servomechanism motor are completely unknown. A second order ARMA model described in (3) is utilized in this experiment. The aim is to identify the unknown parameters of the DC motor recursively, and at each time instance tune the control gains according to (22). Here, a 5-bit PRBS is injected to excite the control loop for proper parameter estimation and improve the convergence rate with PRBS amplitude $\triangle \mathrm{PRBS}=$ 0.3. Importantly, this disturbance only exists at the identification process. From the measured data, the ERLS performs the cycle-by-cycle parameter estimation algorithm previously described to identify the parameters of the DC motor and minimize the estimation error signal. The practicalbased results show that the ERLS algorithm successfully identifies the discrete model of the servomotor (Fig. 8), thus, the estimated error signal converges to minimum value. At each sample instance, the pole-placement control coefficients, (22) are tuned adaptively based on the real time estimation of the system parameters of the servomotor. The tracking performance and the transient characteristics of the servomotor are determined by applying a position step change in the reference signal. The results shown in Fig. 9, demonstrate that the servomotor track the change into position successfully with superior transient performance. The PRBS is constantly injecting into the closed loop and superimposed with control action signal; this disturbance is clearly shown in the position output (see Fig. 9). Again, this disturbance only appears during identification process.

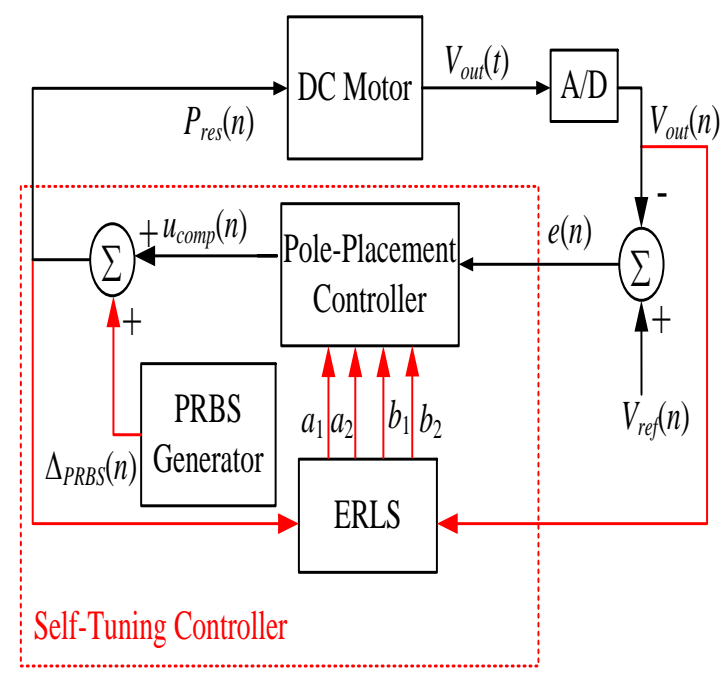

(a) 


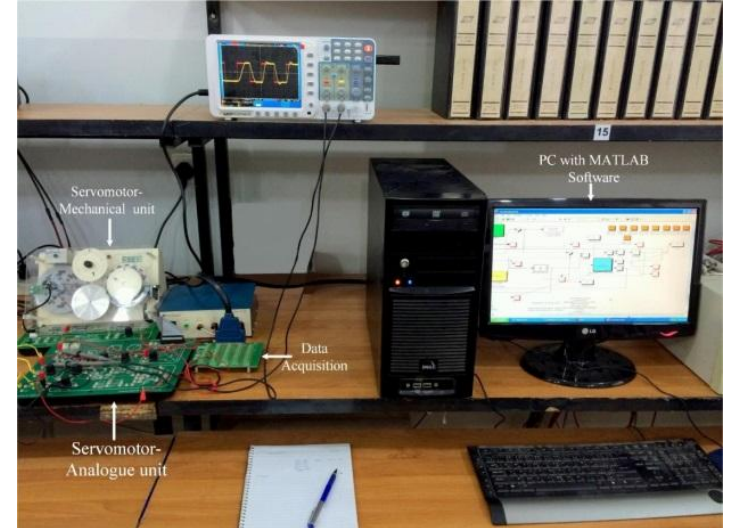

(b)

Fig.7. (a): Proposed self-tuning controller scheme. (b): Hardware system setup.

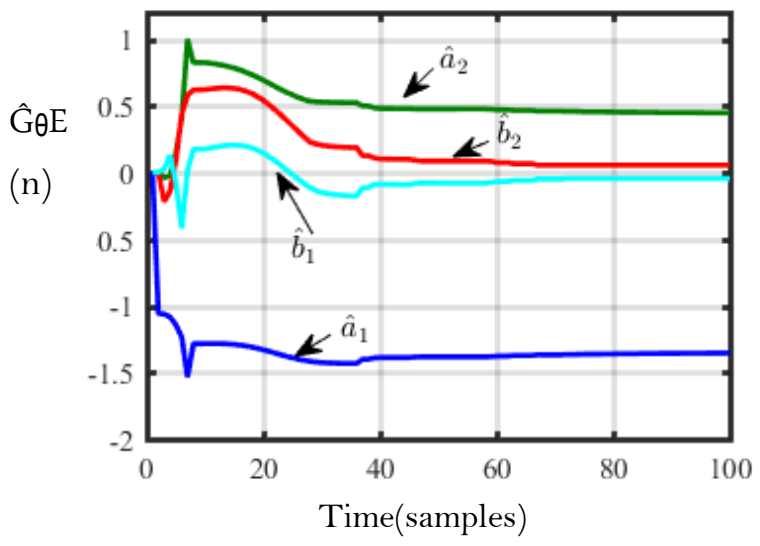

Fig.8. Experimental parameters estimation of DC Motor

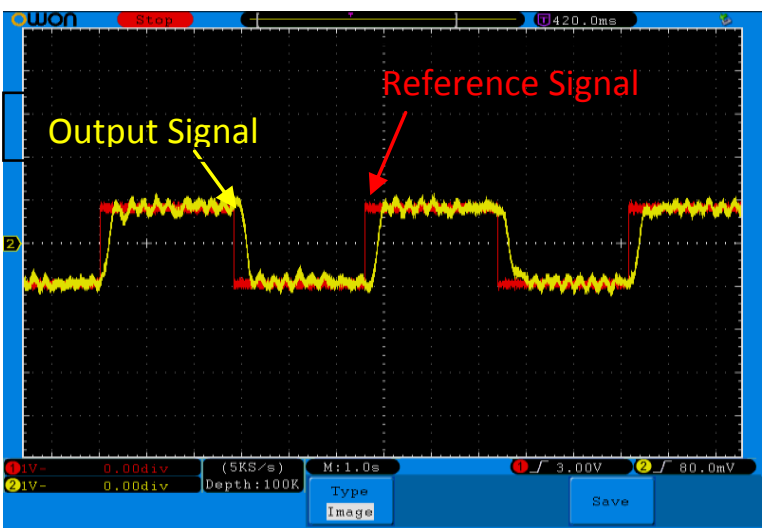

Fig.9. Transient response of self-tuning controller at $\triangle P R B S=0.3 \mathrm{~V}$.

\section{CONCLUSION}

This paper has presented a real time self-tuning controller based on pole placement scheme. The developed paradigm has tested and validated on the DC motor system for real-time parametric estimation of the system model. Simulation and experimental results demonstrated the effectiveness of the ERLS technique, where it provides promising results in terms of convergence rate and accurate parameters estimation, as well as it can be simply integrated with many adaptive and self-tuning controllers. In addition, results shown that the pole placement adaptive controller obtained a very good dynamic performance but on expenses of hardware cost, where the computation burden of the overall scheme is high. Therefore, more investigation should be carried out to reduce the computation complexity of the self-tuning controller. This can be achieved by examining different estimation algorithms to further reduce the computation complexity, as well as by integrating a more hardware efficient controller. Ongoing research also focusing on embedded implementation of the proposed tuning controller based on low-cost digital signal processor.

\section{REFERENCES}

[1] G. Moleykutty, "Speed control of separately excited DC motor," American journal of applied sciences, 2008.

[2] T. Gundogdu and G. Komurgoz, "Adaptive PID controller design by using adaptive interaction approach theory," in Electric Power and Energy Conversion Systems (EPECS), 2013 3rd International Conference on, 2013, pp. 1-5

[3] M. Algreer, M. Armstrong, and D. Giaouris, "Adaptive PD+I Control of a Switch-Mode DC-DC Power Converter Using a Recursive FIR Predictor," IEEE Transactions on Industry Applications, vol. 47, pp. 21352144, 2011.

[4] B. M. Mohan and S. Arpita, "Analytical Structures for Fuzzy PID Controllers?," IEEE Transactions on Fuzzy Systems, vol. 16, pp. 52-60, 2008.

[5] A. Kiam Heong, G. Chong, and L. Yun, "PID Control System Analysis, Design, and Technology," IEEE Transactions on Control Systems Technology, vol. 13, pp. 559-576, 2005.

[6] D. Xiao-Gang, D. Hua, and L. Han-Xiong, "A Saturation-Based Tuning Method for Fuzzy PID Controller," IEEE Transactions on Industrial Electronics, vol. 60, pp. 5177-5185, 2013.

[7] M. Algreer, M. Armstrong, and D. Giaouris, "System Identification of PWM DC-DC Converters During Abrupt Load Changes," in IEEE Industrial Electronics Conference (IECON 2009), 2009, pp. 1788-1793.

[8] A. D. G. Hyalij, P. Shendge, and B. Patre, ""Real Time Implementation of Time Delay Controller for DC Motor Speed Control,"" International Journal of Recent Trends in Engineering, pp. pp. 353-358, 2009.

[9] Y. Jianyong, J. Zongxia, and M. Dawei, "Adaptive Robust Control of DC Motors With Extended State Observer," IEEE Transactions on Industrial Electronics, vol. 61, pp. 3630-3637, 2014.

[10] C.-M. Lin, L. Chih-Min, and C. Chun-Wen, "SoPCBased Adaptive PID Control System Design for Magnetic Levitation System," IEEE Systems Journal, vol. 5, pp. 278-287, 2011.

[11] R. Shanmugasundram, K. M. Zakariah, and N. Yadaiah, "Implementation and Performance Analysis of Digital Controllers for Brushless DC Motor Drives," IEEE/ASME Transactions on Mechatronics, vol. 19, pp. 213-224, 2014.

[12] A. Rubaai and P. Young, "EKF-Based PI-/PD-Like Fuzzy-Neural-Network Controller for Brushless Drives," IEEE Transactions on Industry Applications, vol. 47, pp. 2391-2401, 2011.

[13] F. B. S. Zouari, Kamel; Benrejeb, Mohamed, "Adaptive Internal Model Control of a DC Motor Drive System 
Using Dynamic Neural Network " Journal of Software Engineering \& Applications;Mar2012, Vol. 5 Issue 3, p168, 2012.

[14] J.-f. Xiao, L. Zhang, M. Ou, and F.-h. Zhu, "BLDC motor field orientation control system based on LPIDBP neural network," in IET International Conference on Information Science and Control Engineering 2012 (ICISCE 2012), 2012, pp. 1-4.

[15] J. Fang, X. Zhou, and G. Liu, "Instantaneous torque control of small inductance brushless DC motor," IEEE Transactions on Power Electronics, vol. 27, pp. 49524964, 2012.

[16] R.-J. Wai and R. Muthusamy, "Fuzzy-neural-network inherited sliding-mode control for robot manipulator including actuator dynamics," IEEE Transactions on Neural Networks and Learning Systems, vol. 24, pp. 274-287, 2013.

[17] L. Yang, Z. Jin, X. Mingzi, and L. Hui, "Model Reference Adaptive Control-Based Speed Control of
Brushless DC Motors With Low-Resolution Hall-Effect Sensors," IEEE Transactions on Power Electronics,, vol. 29, pp. 1514-1522, 2014.

[18] M. A. Khanesar, O. Kaynak, and M. Teshnehlab, "Direct Model Reference Takagi-Sugeno Fuzzy Control of SISO Nonlinear Systems," IEEE Transactions on Fuzzy Systems, vol. 19, pp. 914-924, 2011.

[19] F. Golnaraghi and B. C. Kuo, Automatic Control Systems, 9th ed.: John Wiley \& Sons. Inc., 2009.

[20] S. S. Haykin, Adaptive Filter Theory, 4th ed. Upper Saddle River, NJ: Prentice Hall, 2002.

[21] J. B. V.Bobal, J.Fessl, and J.Machacek, Digital Selftuning Controllers: Algorithms, Implementation and Applications: Springer-Verlag London limited 2005.

[22] A. Kelly and K. Rinne, "Control of DC-DC Converters by Direct Pole Placement and Adaptive Feedforward Gain Adjustment," in IEEE Applied Power Electronics Conference and Exposition (APEC 2005), 2005, pp. 1970-1975 Vol. 3. 